R. J. Cohen and W. T. Sullivan, III, eds.

\title{
Radio Interference Monitoring and Databases
}

\author{
W. van Driel \\ Unité Scientifique Nançay, Observatoire de Paris, France
}

\begin{abstract}
For astronomers, monitoring of the radio spectrum is an obvious necessity in order to do observations which are free of interfering signals. Recent practice has shown that the installation of dedicated RFI surveillance antennas at radio astronomy sites has virtually become a necessity. Data obtained with such antennas have carried significant weight in discussions with other radio spectrum users. Furthermore, the growing number of dedicated antennas for RFI surveillance at radio observatories has opened the possibility for the establishment of common RFI databases. Such databases can provide objective numbers showing the degradation of the effective use of protected frequencies due to interference, to be used by astronomers as well as by their partners in frequency protection. As it is all too easy to drown in such a sea of data, discussions between astronomers themselves and with their partners are clearly necessary to define the form, implementation and use of such databases.
\end{abstract}

\section{RFI Monitoring}

Frequency bands allocated to the Radio Astronomy Service (RAS) - even primary allocations - clearly are increasingly threatened. This is seen in the everyday experiences of astronomers world-wide and in the proposals by active Services for further frequency allocations in bands shared with astronomy or in adjacent bands. Clearly, radio astronomy needs to protect itself, to keep its allocated bands clean and useable for the scientific research for which they were allocated. A crucial step in the complex process of frequency protection is the organised and objective monitoring of our allocated bands for interfering signals (see also R. Fisher, this Volume).

In order to see how much astronomical observations are suffering from radio interference (RFI, or EMI), and to study its increase in time, we first need to define the "pain" in an objective way so we know what to measure. The International Telecommunication Union (ITU) has provided a handle on this aspect with its definition of the level of interference considered "harmful" to radio astronomy observations as "... the level which causes an increase of $10 \%$ in the measurement errors, relative to the errors due to system noise alone" (ITU-R Handbook on Radio Astronomy 1995). Note that this means in no way that "radio astronomers should accept the loss of $10 \%$ of their data", as it is sometimes misquoted by other spectrum users.

Radio astronomers need measures of the degradation of their instruments' performance made in a consistent, quantitative and objective way on a regular 
basis. Such RFI data can provide vital evidence in case of conflicts with other spectrum users, when rulings or arbitration are called for. In a strictly legal sense, only observations made at official monitoring stations are acceptable in spectrum regulation matters; in practice, however, observations made at radio astronomy sites of violations of protected bands have been proven to be very useful as well.

In practice, RFI monitoring data can be obtained in several ways:

- Monitoring by monitoring stations of regulatory authorities, like those in Leeheim (Germany) and Baldock (UK) in Europe. These stations can be used to monitor both the main, side-band and spurious emissions of targetted emitters, including satellites. Disadvantages of the presently operating official tracking antennas are their size (about 10 meter diameter), small compared to the size of radio astronomy telescope dishes, and the heavy demand made on them for monitoring all sorts of emitting sources all over the spectrum, limiting the time they can be allocated to monitor RFI in threatened RAS bands. An advantage is their fast tracking capability, allowing on-source integrations of fast-moving Low Earth Orbiting (LEO) satellites. Also, their use is not free of charge, so in case of conflict the question is who pays, the polluter or the victim service?

- Large radiotelescopes themselves can also be used for RFI monitoring. Their advantage is their sensitivity, as these are very large antennas - the largest, Arecibo (Puerto Rico), has a diameter of 300 meter while 100-meter class instruments are in operation at Effelsberg (Germany), Green Bank (Virginia, U.S.A.) and Nançay (France). Their disadvantage is their inability to measure the location and main frequency of an interfering signal, crucial for the determination of the polluter. In fact, their sensitivity makes them virtually omnidirectional RFI detectors, as even their far sidelobes can pick up interfering signals. Also, they are very sensitive to side-band and spurious emissions of emitters in adjacent frequency bands, in which they are not observing, so much so that sometimes they cannot measure the main frequency of the interfering signal (because the sensitive receivers saturate). On the observer's monitoring console these sidelobe-detected, out of band interfering signals are simply seen mixed with the main beam signal of the targetted astronomical source.

- Dedicated RFI monitoring antennas have already been put into operation by a number of radio astronomy observatories. The disadvantage of the systems presently in use is their small size (a few meters in diameter at most) and their inability to track fast LEO satellites, but their advantage is the operation around the clock by the victim service, radio astronomy itself. They can be used for regular monitoring of known sources of interference (such as radars, satellites) as well as searches for interfering signals, either as a general RFI surveillance programme or as a rapid reaction response to an alert given by the site's main radio telescopes.

An example is the Nançay Surveillance Antenna (NSA) operating in central France (see Figure 1). Since 1988, after the launch of the notorious GLONASS satellites, regular RFI monitoring has been carried out at Nançay. The system was mounted on a $22-\mathrm{m}$ high tower in 1992 to allow its unattenuated operation above treetop level, as the Nançay Observatory site lies in the great Sologne forest. The NSA system has two steerable antennas, covering the $100-500 \mathrm{MHz}$ 


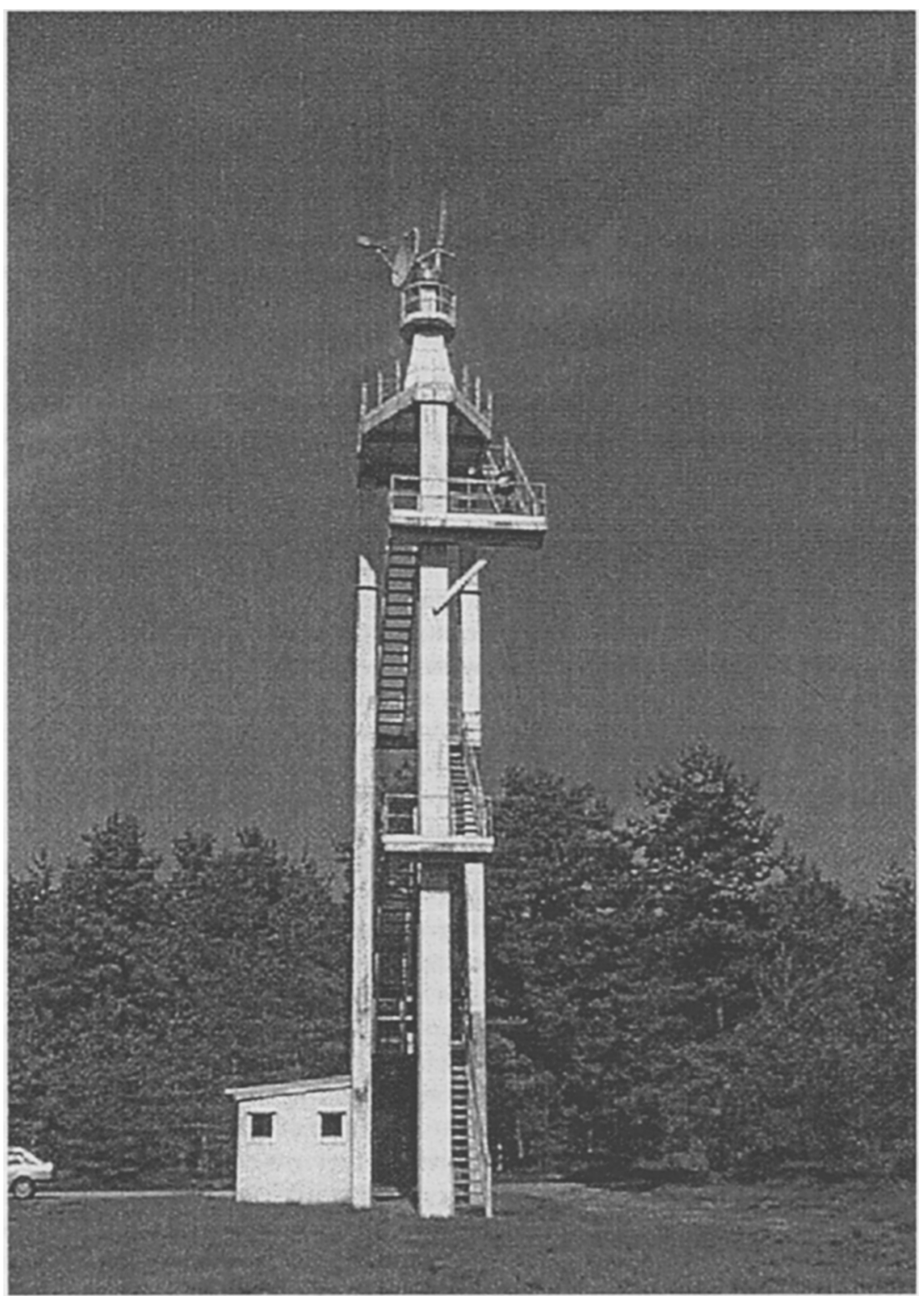

Figure 1. An example of a dedicated RFI monitoring antenna at a radio astronomy observatory, the Nançay Surveillance Antenna (NSA), mounted on a $22-\mathrm{m}$ high tower. The $1.8-\mathrm{m}$ diameter parabola is used for RFI monitoring in the $1-3.5 \mathrm{GHz}$ range; the log-periodic antenna covers the $100-500 \mathrm{MHz}$ range. 
and 1-3.5 GHz frequency ranges corresponding to, respectively, the frequency coverage of the site's radioheliograph and decimetric telescope.

For RFI monitoring at radio observatories, whether performed with a dedicated monitoring system or picked up with the main telescopes, the registration of the occurrence of RFI is a task for the personnel responsible for the execution of the observations (in general technical observatory staff) rather than for the astronomers whose scientific programs are being executed, as the latter often recover their data later at their own Institute through the Internet.

As mentioned above, the principal goal of RFI monitoring is the measurement of the degradation of the data quality, for which the ITU Regulations provide guidelines. For an effective handling of a potential avalanche of data, automatized RFI recognition and characterisation algorithms listing the relevant parameters of the detected RFI (see below) need to be developed and made available to the astronomical community, recognizing the need to provide homogeneous data to be accumulated into international databases.

\section{RFI Databases}

Once the Radio Astronomy Service has access to a regular stream of homogeneous RFI monitoring data, whether through official monitoring stations or through its own operations, it is extremely useful to assemble this input into databases. An obvious caveat is that one can easily drown in such a sea of data, while no useful product or conclusion will emerge from our efforts to help protect our allocated frequencies. Our partners in protection, namely the Agencies charged with national frequency allocation, international regulatory organisations and mediators, need clear, objective measured numbers to be able to assist us and to decide on our future access to the Universe.

Though an RFI database can be extremely useful for an observatory in its struggle to keep its local environment clean, gathering data from sites on a national or international level is an obvious goal, as it provides a powerful tool for the Service in frequency protection. In a European framework, a database concept has been established by CRAF (the Committee on Radio Astronomy Frequencies of the European Science Foundation) in its effort to gather information on the RFI situation and its evolution. Recent negotiations on the continued use of the $1610.6-1613.8 \mathrm{MHz}$ band by CRAF, representing all European observatories, for the protection of one of radio astronomy's primary allocations, have shown the strength of international solidarity among observatories and the added value of sharing RFI monitoring data.

The format of the CRAF database is given in Table 1 ; it contains basic elements needed to quantify a recorded RFI event. The first version of an accompanying software package has also been developped for interrogating the database, which can provide data on

- the frequency of occurrence as function of time (year, month, weekday, hour)

- the percentage of data lost

- the periodicity of a particular interference 
- the comparison between different antennas

For further information, see the CRAF Website http://www.astron.nl/craf/

Table 1. CRAF RFI database parameters

\begin{tabular}{|c|c|c|c|}
\hline Field & Field_name & $\begin{array}{r}\text { Width } \\
\text { (in bytes) }\end{array}$ & Format description \\
\hline 1 & DATE & 8 & yy-mm-dd \\
\hline 2 & STATION & 10 & up to 10 characters \\
\hline 3 & START & 5 & hh:mm (UT) \\
\hline 4 & END & 5 & hh:mm (UT) \\
\hline 5 & ANTENNA & 4 & (according coding) \\
\hline 6 & RFIFREQ & 10 & EMI bandwidth in $\mathrm{MHz}$ (accuracy \\
\hline 7 & BANDWIDTH & 10 & $\begin{array}{l}\text { EMI bandwidth in MHz (accuracy } \\
0.001 \mathrm{MHz} \text { - format is fffff.fff }\end{array}$ \\
\hline 8 & REP_INTERVAL & 4 & $\begin{array}{l}\text { Repetition interval of pulses } \\
\text { (for radars): seconds }\end{array}$ \\
\hline 9 & INTENSITY & 6 & Intensity of interference \\
\hline 10 & INT_UNIT & 2 & Intensity unit: $\mathrm{KE}=$ Kelvin, $\mathrm{JY}=\mathrm{J}$ ansky \\
\hline 11 & RFI_AZ & 3 & Azimuth of EMI source (if available) \\
\hline 12 & RFIEL & 3 & Elevation of EMI source (if available) \\
\hline 13 & TYPE & 2 & $\begin{array}{l}\text { kind of observation: } \mathrm{BR}=\text { broadband } \\
\mathrm{SP}=\text { spectral }\end{array}$ \\
\hline 14 & ANT_AZ & 3 & Azimuth of observations in degrees \\
\hline 15 & ANT_EL & 3 & Elevation of observations in degrees \\
\hline 16 & DEG & 3 & Degree of degradation in percent \\
\hline \multirow[t]{2}{*}{20} & Reserved & 1 & \\
\hline & Total & 80 & bytes \\
\hline
\end{tabular}

In the course of gathering information on RFI in its own protected bands, the radio astronomy service needs to observe outside these bands as its telescope are very sensitive to out of band emission. Thus, information can also be accumulated on spectrum occupancy in other bands. It is clear that such data are liable to non-disclosure, as they cover military and commercial uses. Therefore, access to databases on RFI for radio astronomy, such as the one operated by CRAF, needs to remain restricted.

\section{References}

ITU-R Handbook on Radio Astronomy, 1995, Radiocommunications Bureau, International Telecommunication Union, Geneva 